\section{Niedrig dosiertes II-2 beim systemischen Lupus erythematodes}

He J et al. Efficacy and safety of low-dose IL-2 in the treatment of systemic lupus erythematosus: a randomised, double-blind, placebo-controlled trial. Ann Rheum Dis 2020; 79: 141-149. doi:

10.1136/annrheumdis-2019-215396

Patienten mit einem systemischen Lupus erythematodes (SLE) erleiden in Folge der immunsuppressiven Therapie gehäuft Infektionen. Erste Untersuchungen deuten darauf hin, dass niedrig dosiertes Interleukin-2 (Il-2) die immunologische Balance wiederherstellt, die Immunantwort auf Infektionen unterstützt und sogar zu einer klinischen Besserung führt. Eine randomisierte Doppelblindstudie prüfte nun die Effektivität und Sicherheit dieser Therapiestrategie.

An der chinesischen Studie nahmen $60 \mathrm{Er}$ wachsene mit einem trotz Standardtherapie aktiven SLE teil. Je die Hälfte der Patienten erhielten zusätzlich zu ihrer Standardtherapie über einen Zeitraum von 12 Wochen niedrig dosiertes II-2 bzw. ein Placebo in Form subkutaner Injektionen. Während dieser Interventionsphase sowie der folgenden 12-wöchigen Nachbeobachtungsphase untersuchten die Studieninitiatoren die Patienten regelmäßig klinisch sowie laborchemisch. Als primären Studienendpunkt definierten die Wissenschaftler das anhand des sogenannten SLE Responder Index-4 (SRI-4) gemessenen Therapieansprechens in Woche 12. Die sekundären Studienendpunkte umfassten unter anderem die Sicherheit der Behandlung sowie die Dynamik verschiedener Immunzell-Subpopulationen, bspw. der T-Zellen und der natürlichen Killerzellen (NK-Zellen).

\section{Ergebnisse}

Das Durchschnittsalter der mit II-2 bzw. Placebo behandelten Patienten betrug 31,6 bzw. 29,8 Jahre und die durchschnittliche Krankheitsdauer 66,7 bzw. 63,6 Monate. 13 bzw. 12 Patienten dieser beiden Gruppen litten an einer Lupusnephritis. 55,2\% der mit niedrig dosiertem II-2 und 30,0\% der mit Placebo behandelten Studienteilnehmer hatten gemäß SRI-4 nach 12 Wochen auf die Behandlung angesprochen ( $p=0,052$; pri- märer Endpunkt nicht erreicht). Nach 24 Wochen betrug die Ansprechrate in den beiden Gruppen 65,5 bzw. 36,7\% ( $p=0,027)$. Im Kollektiv der Patienten mit einer Lupusnephritis erreichten nach 12 Wochen 53,9\% der mit II-2 aber nur 8,3\% der mit Placebo behandelten Studienteilnehmer eine Komplettremission $(p=0,013)$. Gleiches galt für die Auswertung nach 24 Wochen (53,9 vs. $16,7 \% ; p=0,036)$. Schwere Infektionen erlitten 2 Patienten der Kontroll- aber kein Patient der Interventionsgruppe. Die immunologische Analyse ergab: Die niedrig dosierte II-2-Therapie induzierte eine deutliche Expansion der regulatorischen T-Zellen sowie der NK-Zellen.

\section{FAZIT}

Angesichts dieser Studienergebnisse halten die Wissenschaftler niedrig dosiertes II-2 zur Therapie des SLE für effektiv und sicher. Die immunologischen Analysen deuten ihrer Einschätzung nach zudem darauf hin, dass die Behandlung zur Wiederherstellung des immunologischen Gleichgewichts betragen könnte. Weitere große randomisierte kontrollierte Studien müssen diese Ergebnisse nun bestätigen.

Dr. med. Judith Lorenz, Künzell 